\title{
Update on Safety Profiles of Vitamins BI, B6, and BI2: A Narrative Review
}

This article was published in the following Dove Press journal:

Therapeutics and Clinical Risk Management

\section{Carlos-Alberto Calderon- Ospina (iD ${ }^{\prime} *$ Mauricio Orlando Nava- Mesa iD ${ }^{2, *}$ \\ Ana María Paez-Hurtado (iD) ${ }^{2}$ \\ 'Center for Research in Genetics and Genomics (CIGGUR), GENIUROS Research Group, School of Medicine and Health Sciences, Universidad del Rosario, Bogotá, Colombia; ${ }^{2}$ Neuroscience Research Group (NEUROS), School of Medicine and Health Sciences, Universidad del Rosario, Bogotá, Colombia}

*These authors contributed equally to this work
Correspondence: Carlos-Alberto Calderon-Ospina

School of Medicine and Health Sciences, Universidad del Rosario, Carrera 24 No. 63C-69, Bogota, DC, Colombia

Tel +57I-2970200 Ext 33 I8

Fax +57I-28I8583

Email carlos.calderon@urosario.edu.co

\begin{abstract}
The neurotropic B vitamins B1 (thiamine), B6 (pyridoxine), and B12 (cobalamin) are essential for proper functioning of the nervous system. Deficiencies may induce neurological disorders like peripheral neuropathy $(\mathrm{PN})$ and mainly occur in vulnerable populations (eg, elderly, diabetics, alcoholics). As epidemiologic cohort studies raised safety concerns about vitamin B6/B12 intake being potentially associated with increased risks of hip fracture (HF) and lung cancer (LC), we explored these aspects and performed comprehensive literature searches. However, we suggest not to neglect actual high-risk factors (eg, smoking in LC, higher age in HF) by focusing on individual nutrients, but to examine the complex interaction of numerous factors involved in disease development. Because it warrants continued consideration, we also provide an update on neurotoxicity associated with vitamin B6. We consider that neurological side effects due to vitamin B6 intake are rare and only occur with high daily doses and/or longer treatment duration. The benefit-risk ratio of highdose treatment with neurotropic B vitamins in indications like PN is therefore considered advantageous, particularly if dosing recommendations are followed and serum levels monitored.
\end{abstract}

Keywords: neurotropic B vitamins, safety, thiamine, pyridoxine, cobalamin, neurotoxicity, hip fracture, lung cancer

\section{Introduction}

Neurotropic B vitamins are essential for proper functioning of the nervous system. ${ }^{1-3}$ Deficiencies possibly leading to neurological disturbances, such as peripheral neuropathy (PN), may be due to an unbalanced diet (decreased availability) but mainly occur in certain vulnerable populations (eg, elderly, diabetics, alcoholics, dialysis patients, patients with gastrointestinal diseases) due to impaired absorption/utilization, accelerated usage, increased loss ${ }^{4-13}$ or increased hydrolysis during inflammatory diseases. ${ }^{14}$ In addition, deficits may be related to the chronic use of several widely used medications (eg, omeprazole, methotrexate, metformin). ${ }^{15-17}$ The use of B vitamins, particularly vitamins B1 (thiamine), B6 (pyridoxine), and B12 (cobalamin), for the treatment of PN, is well-established worldwide $^{18-24}$ and usually considered safe, even with high doses and in the long term. $^{19}$

In rare cases, vitamin B12 administration can be associated with dermal adverse effects (eg, acne). ${ }^{25}$ The risk appears to be higher with hydroxocobalamin than cyanocobalamin. So far, the European Food Safety Authority (EFSA) reported dermal reactions mainly with high parenteral doses $(12 \times 5 \mathrm{mg}){ }^{26}$ The authors of a recent case report series attributed acne eruption in one of their patients to oral 
vitamin B12 administration, but the patient took a multivitamin preparation containing vitamins B1, B2, $\mathrm{B} 6$, and B12 (no doses specified). ${ }^{25}$ Acne due to vitamin B12 treatment usually resolves completely after treatment cessation. $^{26-29}$ Apart from dermal reactions, cumulative adverse effects of vitamin B12 high-dose and long-term treatment are unknown. Several randomized placebocontrolled trials provide evidence on long-term use of high-dose vitamin $\mathrm{B} 12$ by oral route for up to 7 years without mentioning signs of overdosage or reporting sideeffects different from placebo. ${ }^{30-32}$

Low dose intake of neurotropic B vitamins according to Recommended Dietary Allowance (RDA) to prevent deficiencies in the healthy population is well-established and generally considered safe for long-term use; ${ }^{25}$ there is rarely any new safety information available. However, over the past few years, some epidemiologic cohort studies raised new safety concerns about $\mathrm{B}$ vitamins (notably vitamins B6 and B12) being associated with an increased risk of hip fracture and lung cancer. ${ }^{33,34}$ In addition, neurological adverse effects (such as ataxia and sensory neuropathy) under long-term/high-dose treatment with vitamin B6 warrant continued consideration worthy of being updated, although they are well-described and usually reversible. ${ }^{1,25}$ Therefore, we aimed to explore and update these three aspects - neurotoxicity, hip fracture risk, and lung cancer risk - in the present narrative review with the help of comprehensive literature searches.

\section{Methods}

\section{Literature Search and Selection Strategy}

We performed systematic literature searches in PubMed (https://www.ncbi.nlm.nih.gov/pubmed) and Cochrane (https://www.cochrane.org/) for English-language articles with available full text that had been published through October 2, 2019 (hip fracture, lung cancer) or October 30, 2019 (neurotoxicity). The search terms were combinations of "vitamin B1", "thiamine", "vitamin B6", "pyridoxine", "vitamin B12", "cobalamin", OR "B vitamins" AND "hip fracture" OR "lung cancer" as well as combinations of "vitamin B6" OR "pyridoxine" AND "neurotoxicity" OR "neurotoxic". Additional articles were identified through manual searches in Google Scholar and the authors' own files. Figure 1 shows the literature search and selection strategy. To receive a comprehensive overview, we did not filter for original research articles and also considered reviews, but did not include reviews in the overview
(Tables 1-3) that list selected original articles (randomized controlled trials [RCTs], cohort studies, case reports). In order to not miss any relevant, not properly indexed articles in PubMed, we also did not filter for "humans", although we excluded original articles on animal research.

\section{Current Evidence and Discussion Vitamin BI and Adverse Reactions}

We were unable to identify any relevant articles for vitamin B1 (thiamine) regarding the selected safety aspects, and - to the best of our knowledge - there are no reports of adverse effects from oral thiamine consumption by food or supplements. Doses of thiamine $>5 \mathrm{mg}$ decline its absorption rate and the excess is excreted in the urine. ${ }^{25}$ In contrast to the oral route, parenteral use has been associated with phlebitis and very rarely with hypersensitivity reactions such as pruritus, respiratory distress, nausea, abdominal pain, and even anaphylactic shock, which are mainly associated with the administration route $(<2 \%)^{35-37}$

\section{Vitamin B6 and Neurotoxicity}

The neurotoxic effect of ultrahigh-dose vitamin B6 (1,000-7,000 $\mathrm{mg} / \mathrm{kg}$ ) has been known since the 1940s from animal experiments. ${ }^{25}$ As reviewed by Rao et al, ${ }^{38}$ toxic exposure to vitamin B6 induces primary injury of nerve cell bodies (neuronopathy), possibly followed by necrosis as well as axonal and myelin degeneration. If the injury is not too advanced, the nerves can regenerate after treatment cessation, resulting in clinical improvement or resolution of symptoms. ${ }^{38}$ Relevant original articles on neurotoxicity of vitamin B6 in humans obtained by our searches included seven case reports/series, one prospective study, and one case-control study (Table 1). ${ }^{39-, 47}$ In half of them, neurotoxic side-effects of vitamin B6 were mostly due to very high doses and/or long-term treatment and resolved after treatment cessation. ${ }^{39-41}$ Specifically, reported clinical neuropathy cases occurred in a timeframe of 2-24 months depending on the administered dose: after 12 months or longer with doses of $\leq 2$,$000 \mathrm{mg} /$ day, and after less than 12 months with doses $>2,000 \mathrm{mg} /$ day. $^{39-46}$ The first clinical assessment study of pyridoxine-induced neurotoxicity in humans by Schaumburg et $\mathrm{al}^{47}$ included seven patients with severe sensory neuropathy in the extremities after taking 2,000-$6,000 \mathrm{mg} /$ day pyridoxine for $2-40$ months. Four of these individuals were not able to walk. All symptoms, 


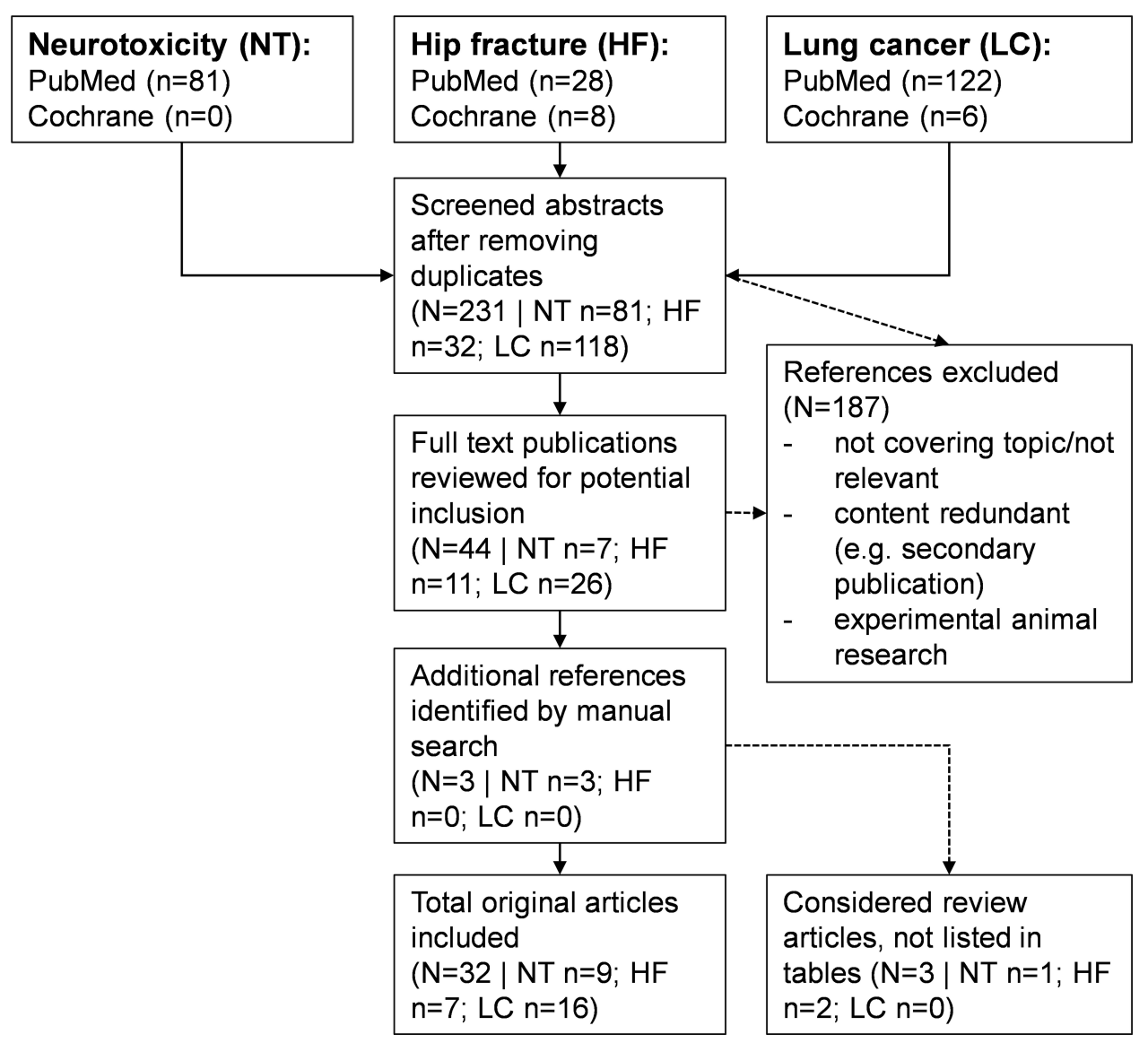

Figure I Literature search and selection strategy for the following adverse events possibly associated with the use of B vitamins: neurotoxicity (NT), hip fracture (HF), and lung cancer (LC). $n=$ size number.

assessed through objective neurological diagnosis, improved after medication discontinuation. ${ }^{47}$ Another case series report by Parry and Bredesen ${ }^{46}$ studied 16 patients with neuropathy (all assessed by history and examination, seven electrophysiologically confirmed diagnoses, two sural nerve biopsies) after taking 100-5,000 mg/day pyridoxine for up to 72 months. Patients with doses $\leq 500 \mathrm{mg} /$ day showed neuropathy after 23 months on average, while those taking 5,$000 \mathrm{mg} /$ day developed symptoms on average after around 3 months. In contrast, none of the patients taking $50 \mathrm{mg} /$ day for $<6$ months presented with neuropathy, but when the intake exceeded 6 months, 20\% developed symptoms. $^{46}$ These results indicate an association between neuropathy and dose/duration of vitamin B6 use, suggesting that $50 \mathrm{mg}$ /day for $<6$ months is safe. As here, in the publications describing cases with persisting symptoms after vitamin B6 discontinuation (Table $1),{ }^{42-45}$ it was unclear whether the neurological symptoms were only attributable to vitamin B6 treatment or were favored by underlying diseases or co-medication. The study from Dalton and Dalton ${ }^{41}$ deserves special attention because it is usually cited when the potential neurotoxicity of low doses such as $50 \mathrm{mg}$ is discussed. ${ }^{25}$ However, the study design has often been criticized, for example by the Food and Nutrition Board of the Institute of Medicine (IOM), because neurological symptoms were not adequately detailed and actual doses may have been underestimated due to parallel intake of vitamin supplements; thus, the study appears unsuitable for determining the upper limit. ${ }^{35}$

Van Hunsel et $\mathrm{al}^{42}$ described 90 assumed adverse drug reactions (ADRs) in the form of neuropathy reported to the Netherlands Pharmacovigilance Center between 1991 and 2017 that had been attributed to the intake of vitamin B6 supplements. In the description of selected cases, they also mentioned very few permanent cases. However, the causality between vitamin B6 intake and the occurrence of ADRs was not always clear, and important details on many cases were missing (eg, exact vitamin B6 amount, 
Table I Characteristics of Included Studies and Case Reports on Neurotoxicity of Vitamin B6

\begin{tabular}{|c|c|c|c|c|}
\hline Reference & $\begin{array}{l}\text { Study } \\
\text { Type and } \\
\text { Country }\end{array}$ & Sample Size & Main Findings & Comment \\
\hline $\begin{array}{l}\text { Echaniz-Laguna } \\
\text { et al, } 2018^{39}\end{array}$ & $\begin{array}{l}\text { Case } \\
\text { report, } \\
\text { France }\end{array}$ & $\begin{array}{l}\mathrm{N}=\text { I patient with } \\
\text { homocystinuria }\end{array}$ & $\begin{array}{l}\text { Patient treated with vitamin B6 } \\
\mathrm{I}, 250-1,750 \mathrm{mg} / \text { day for } 20 \text { years } \\
\text { developed progressive sensory } \\
\text { neuropathy with ataxia and impaired } \\
\text { sensation in the extremities; reduction } \\
\text { of vitamin B6 dosage to } 500 \mathrm{mg} / \text { day led } \\
\text { to disappearance of symptoms }\end{array}$ & $\begin{array}{l}\text { Very high-dose long-term treatment } \\
\text { led to symptoms that were still } \\
\text { reversible }\end{array}$ \\
\hline $\begin{array}{l}\text { van Hunsel } \\
\text { et al, } 2018^{42}\end{array}$ & $\begin{array}{l}\text { Case series, } \\
\text { Netherlands }\end{array}$ & $\begin{array}{l}\mathrm{N}=90 \text { ADR reports on products } \\
\text { containing vitamin B6 with at } \\
\text { least one ADR being "peripheral } \\
\text { neuropathy" }\end{array}$ & $\begin{array}{l}\text { Amount of vitamin B6 varied } \\
\text { between I. } 4 \text { and } 100 \text { mg per tablet. } \\
\text { The serum vitamin B6 level was only } \\
\text { known in } 36 \text { cases (mean: } 907 \mathrm{nmol} / \\
\text { L); causality assessment showed it is } \\
\text { plausible for the vitamin B6 } \\
\text { supplements to have caused } \\
\text { complaints such as neuropathies, } \\
\text { especially with higher dosages and } \\
\text { prolonged use, but dosages }<50 \mathrm{mg} / \\
\text { day also cannot be excluded }\end{array}$ & $\begin{array}{l}\text { Case series has several limitations } \\
\text { (see Vitamin B6 and neurotoxicity) }\end{array}$ \\
\hline $\begin{array}{l}\text { Kulkantrakorn, } \\
2014^{43}\end{array}$ & $\begin{array}{l}\text { Case series, } \\
\text { Thailand }\end{array}$ & $\begin{array}{l}\mathrm{N}=3 \text { patients with chronic } \\
\text { underlying diseases (lacunar } \\
\text { infarction, DM, etc.) }\end{array}$ & $\begin{array}{l}\text { Patients consumed a vitamin B6 dose } \\
\text { of } 600 \mathrm{mg} / \text { day for 3-10 years; blood } \\
\text { levels of PLP were } 67-105 \text { times } \\
\text { upper normal; signs of } \\
\text { polyneuropathy and sensory ataxia; } \\
\text { patients showed no significant } \\
\text { improvement of neuropathy and gait } \\
2 \text { years after vitamin discontinuation }\end{array}$ & $\begin{array}{l}\text { High-dose long-term treatment; } \\
\text { authors do not exclude other causes } \\
\text { of neuropathy, but considered statin } \\
\text { therapy and diabetes less likely as } \\
\text { significant factors in two of the } \\
\text { patients due to short duration of } \\
\text { low-dose statin therapy and only I-2 } \\
\text { years of DM (well controlled) }\end{array}$ \\
\hline $\begin{array}{l}\text { Mikalunas et al, } \\
200 \mathrm{I}^{44}\end{array}$ & $\begin{array}{l}\text { Case series, } \\
\text { US }\end{array}$ & $\mathrm{N}=6$ patients on $\mathrm{HPN}$ & $\begin{array}{l}\text { Patients received multivitamin } \\
\text { solution (contained, among other } \\
\text { vitamins, } 4 \mathrm{mg} \mathrm{B6} \text { ); two of the patients } \\
\text { (both with renal failure) had elevated } \\
\text { vitamin B6 blood levels and possible } \\
\text { neurotoxicity (peripheral neuropathy, } \\
\text { involuntary movements); parenteral } \\
\text { multivitamin administration was } \\
\text { stopped in one and decreased to once } \\
\text { weekly in the other; vitamin B6 levels } \\
\text { fell to normal; no improvement in } \\
\text { neurological symptoms }\end{array}$ & $\begin{array}{l}\text { Comorbidities may have contributed } \\
\text { to the neurological symptoms in } \\
\text { these severely ill patients (excessive } \\
\text { levels of water-soluble vitamins have } \\
\text { been reported in patients with renal } \\
\text { failure); periodic monitoring of } \\
\text { serum levels is recommended }\end{array}$ \\
\hline $\begin{array}{l}\text { Berger et al, } \\
1992^{40}\end{array}$ & $\begin{array}{l}\text { Prospective } \\
\text { study, US }\end{array}$ & $N=5$ healthy volunteers & $\begin{array}{l}\text { Volunteers received I or } 3 \mathrm{~g} / \text { day } \\
\text { vitamin B6 over } 1.5-7 \text { months (until } \\
\text { neuropathy symptoms occurred); } \\
\text { high-dose subjects became } \\
\text { symptomatic earlier than low-dose } \\
\text { subjects; clear dose-dependent } \\
\text { relationship; all symptoms gradually } \\
\text { resolved after treatment } \\
\text { discontinuation }\end{array}$ & $\begin{array}{l}\text { Symptoms were reversible despite } \\
\text { very high doses }\end{array}$ \\
\hline
\end{tabular}

(Continued) 
Table I (Continued).

\begin{tabular}{|c|c|c|c|c|}
\hline Reference & $\begin{array}{l}\text { Study } \\
\text { Type and } \\
\text { Country }\end{array}$ & Sample Size & Main Findings & Comment \\
\hline $\begin{array}{l}\text { Albin et al, } \\
1987^{45}\end{array}$ & $\begin{array}{l}\text { Case series, } \\
\text { US }\end{array}$ & $\begin{array}{l}\mathrm{N}=2 \text { patients with mushroom } \\
\text { intoxication }\end{array}$ & $\begin{array}{l}\text { Couple with mushroom intoxication } \\
\text { received an estimated total dose of } \\
132 \mathrm{~g} / \mathrm{I} 83 \mathrm{~g} \text { iv vitamin } \mathrm{B} 6 \text { over } 3 \\
\text { days; developed neurological } \\
\text { symptoms of which some but not all } \\
\text { improved after treatment cessation }\end{array}$ & $\begin{array}{l}\text { Ultra-high doses considerably in } \\
\text { excess even of those recommended } \\
\text { to treat Gyromitra esculenta } \\
\text { intoxication }\end{array}$ \\
\hline $\begin{array}{l}\text { Dalton and } \\
\text { Dalton, } 1987^{41}\end{array}$ & $\begin{array}{l}\text { Case- } \\
\text { control } \\
\text { study, UK }\end{array}$ & $\mathrm{N}=172$ women with PMS & $\begin{array}{l}\text { All women took vitamin B6 ( }<50 \text { to } \\
<500 \mathrm{mg} / \text { day) and had raised serum } \\
\text { levels; I03 women developed } \\
\text { neurological symptoms that resolved } \\
\text { after withdrawal; cases had taken } \\
\text { vitamin B6 for a significantly longer } \\
\text { period than controls ( } 2.9 \pm 1.9 \text { vs } 1.6 \\
\pm 2.1 \text { years) but mean doses were } \\
\text { comparable }\end{array}$ & $\begin{array}{l}\text { Results of the study are considered } \\
\text { unreliable and invalid by } 10 M^{35}\end{array}$ \\
\hline $\begin{array}{l}\text { Parry and } \\
\text { Bredesen, } \\
1985^{46}\end{array}$ & Case series & $\begin{array}{l}\mathrm{N}=16 \text { patients with neuropathy } \\
\text { (all assessed by examination, } 7 \\
\text { by electrophysiological } \\
\text { confirmation and } 2 \text { had sural } \\
\text { nerve biopsy) }\end{array}$ & $\begin{array}{l}62.5 \% \text { of the patients developed } \\
\text { large fiber sensory neuronopathy; } \\
6.4 \% \text { had motor failure and } 31.3 \% \\
\text { showed small fiber involvement. Two } \\
\text { patients had a coasting phenomenon. } \\
\text { Symptoms and clinical improvement } \\
\text { were shown in II patients, several } \\
\text { (not specified) months after } \\
\text { cessation of pyridoxine medication }\end{array}$ & $\begin{array}{l}\text { Small sample size; real pyridoxine } \\
\text { dose intake not clear due to lack of } \\
\text { information on any other vitamin } \\
\text { supplement or medication taking } \\
\text { during the studied time }\end{array}$ \\
\hline $\begin{array}{l}\text { Schaumburg } \\
\text { et al, } 1983^{47}\end{array}$ & Case series & $\mathrm{N}=7$ patients ( 5 women, 2 men $)$ & $\begin{array}{l}\text { Severe sensory neuropathy patients } \\
\text { (showing progressive paresthesia of } \\
\text { both hands and feet, marked sensory } \\
\text { ataxia, absent tendon reflexes and } \\
\text { flexor plantar responses) with high } \\
\text { doses of vitamin B6 } \\
(2,000-6,000 \mathrm{mg} / \text { day) and long-time } \\
\text { intakes ( } 2-40 \text { months) showed } \\
\text { symptom improvement after } \\
\text { cessation of medication }\end{array}$ & $\begin{array}{l}\text { Small sample size; unclear if } \\
\text { symptoms were attributed only to } \\
\text { vitamin B6 intake or to any other } \\
\text { medication }\end{array}$ \\
\hline
\end{tabular}

Abbreviations: ADR, adverse drug reaction; DM, diabetes mellitus; HPN, home parenteral nutrition; IOM, Institute of Medicine; iv, intravenous; PLP, pyridoxal 5' phosphate; PMS, premenstrual syndrome; UK, United Kingdom; US, United States.

exact product composition, actual intake, serum levels, neuropathy-triggering predisposing factors, clinical examinations). Moreover, due to missing data on the number of ADRs in relation to the number of supplement users, the authors could not assess the actual risk of adverse effects at the dose considered the maximal acceptable intake (25 mg). ${ }^{42}$

We conclude that neurological side-effects due to vitamin B6 are rare, habitually reversible, and usually occur after taking high daily doses (>500 mg/day) and/or longer treatment ( $>6$ months).

\section{Vitamin B6 and/or BI2 and Risk of Hip Fracture}

For several years, it has been suggested that high homocysteine (Hcy) levels are associated with lower bone mineral density (BMD), thereby increasing fracture risk. $^{48,49}$ It is also known that B vitamins such as B12 
Table 2 Characteristics of Included Studies on an Association Between Vitamin B6 and/or BI2 Treatment and Hip Fracture Risk

\begin{tabular}{|c|c|c|c|c|}
\hline Reference & $\begin{array}{l}\text { Study Type and } \\
\text { Country }\end{array}$ & Sample Size & Main Findings & Comment \\
\hline Meyer et al, $2019^{33}$ & $\begin{array}{l}\text { Prospective } \\
\text { cohort study, US }\end{array}$ & $\begin{array}{l}N=75,864 \text { postmenopausal } \\
\text { women }\end{array}$ & $\begin{array}{l}\text { Both vitamin } B 6 \text { and } B 12 \text { intake } \\
\text { were associated with increased } \\
\text { fracture risk }\end{array}$ & $\begin{array}{l}\text { Study has several limitations } \\
\text { (see Vitamin B6 and /or BI2 and } \\
\text { risk of hip fracture) }\end{array}$ \\
\hline $\begin{array}{l}\text { Garcia Lopez et al, } \\
2017^{50}\end{array}$ & $\begin{array}{l}\text { Secondary } \\
\text { analysis of } \\
\text { combined data } \\
\text { from two RCTs } \\
\text { (NORVIT, } \\
\text { WENBIT), } \\
\text { Norway }\end{array}$ & $\begin{array}{l}\mathrm{N}=6,837 \text { patients with ischemic } \\
\text { heart disease }\end{array}$ & $\begin{array}{l}\text { Slightly increased risk of hip } \\
\text { fracture for treatment with } \\
\text { vitamin B6 alone }\end{array}$ & $\begin{array}{l}\text { Study has limitations since both } \\
\text { RCTs originally were not } \\
\text { designed or powered to study } \\
\text { hip fractures; baseline data on } \\
\text { BMD, bone markers, falls, etc. } \\
\text { missing }\end{array}$ \\
\hline Dai et al, $2013^{52}$ & $\begin{array}{l}\text { Prospective } \\
\text { population-based } \\
\text { cohort study, } \\
\text { Singapore }\end{array}$ & $\begin{array}{l}\mathrm{N}=63,257 \text { participants from } \\
\text { Singapore Chinese Health Study }\end{array}$ & $\begin{array}{l}\text { Statistically significant inverse } \\
\text { relationship between dietary } \\
\text { vitamin B6 intake and hip } \\
\text { fracture risk in women but not } \\
\text { men; hip fracture risk reduced } \\
\text { by } 22 \% \text { for women in highest } \\
\text { intake quartile compared to } \\
\text { women in lowest intake } \\
\text { quartile; no relation for other } \\
\text { B vitamins of interest }\end{array}$ & $\begin{array}{l}\text { Dietary intake of B vitamins was } \\
\text { recorded using a food- } \\
\text { frequency questionnaire; diet } \\
\text { assessment only at study } \\
\text { recruitment }\end{array}$ \\
\hline $\begin{array}{l}\text { McLean et al, } \\
2008^{53}\end{array}$ & $\begin{array}{l}\text { Longitudinal } \\
\text { follow-up study, } \\
\text { US }\end{array}$ & $\begin{array}{l}\mathrm{N}=1,002 \text { participants from } \\
\text { Framingham Study }\end{array}$ & $\begin{array}{l}\text { Vitamins BI2 and B6 were } \\
\text { inversely associated with hip } \\
\text { fracture risk; low status of } \\
\text { vitamin BI2 and vitamin B6 may } \\
\text { be independent risk factors for } \\
\text { hip fracture }\end{array}$ & $\begin{array}{l}\text { Results may only be valid for } \\
\text { older white men and women; } \\
\text { only plasma concentrations of } \\
\text { B vitamins were available, which } \\
\text { may not provide optimum } \\
\text { assessment of vitamin status }\end{array}$ \\
\hline $\begin{array}{l}\text { Gjesdal et al, } \\
2007^{54}\end{array}$ & $\begin{array}{l}\text { Prospective } \\
\text { cohort study, } \\
\text { Norway }\end{array}$ & $\begin{array}{l}\mathrm{N}=4,766 \text { participants from } \\
\text { Hordaland Homocysteine Study }\end{array}$ & $\begin{array}{l}\text { Hcy seemed to be a predictor of } \\
\text { hip fracture among elderly men } \\
\text { and women while folate was } \\
\text { a predictor among women only; } \\
\text { vitamin BI2 did not predict hip } \\
\text { fracture }\end{array}$ & $\begin{array}{l}\text { Plasma samples for folate and } \\
\text { vitamin } \mathrm{B} 12 \text { determination had } \\
\text { been stored for up to } 10 \text { years } \\
\text { before analysis, which may have } \\
\text { weakened an association }\end{array}$ \\
\hline Morris et al, $2005^{55}$ & $\begin{array}{l}\text { Prospective } \\
\text { cohort study, US }\end{array}$ & $\begin{array}{l}\mathrm{N}=1,550 \text { participants from } \\
\text { NHANES III }\end{array}$ & $\begin{array}{l}\text { Higher serum vitamin BI2 was } \\
\text { related to higher BMD; subjects } \\
\text { with high serum Hcy had } \\
\text { significantly lower BMD than } \\
\text { subjects with low serum Hcy }\end{array}$ & $\begin{array}{l}\text { Results may only be valid for } \\
\text { elderly }\end{array}$ \\
\hline $\begin{array}{l}\text { Lumbers et al, } \\
2001^{56}\end{array}$ & $\begin{array}{l}\text { Case-control } \\
\text { study, UK }\end{array}$ & $\begin{array}{l}\mathrm{N}=75 \text { older female hospital } \\
\text { patients admitted for } \\
\text { emergency surgery of fractured } \\
\text { femur neck and } \mathrm{N}=50 \text { age- } \\
\text { matched independent-living } \\
\text { females }\end{array}$ & $\begin{array}{l}\text { Fracture patients had lower } \\
\text { intakes of vitamin B6 and other } \\
\text { nutrients }\end{array}$ & Small sample size \\
\hline
\end{tabular}

Abbreviations: BMD, bone mineral density; Hcy, homocysteine; NHANES, National Health and Nutrition Examination Survey; NORVIT, Norwegian Vitamin Trial; RCTs, randomized controlled trials; UK, United Kingdom; US, United States; WENBIT, Western Norway B Vitamin Intervention Trial. 
Table 3 Characteristics of Included Studies on an Association Between Vitamin B6 and/or BI2 Treatment and Lung Cancer Risk

\begin{tabular}{|c|c|c|c|c|}
\hline Reference & $\begin{array}{l}\text { Study Type and } \\
\text { Country }\end{array}$ & Sample Size & Main Findings & Comment \\
\hline Takata et al, $2019^{80}$ & $\begin{array}{l}\text { Prospective cohort } \\
\text { study, US }\end{array}$ & $\begin{array}{l}\mathrm{N}=1,064 \text { lung cancer } \\
\text { cases among } 68,236 \\
\text { participants }\end{array}$ & $\begin{array}{l}\text { Neither the use of folic acid-containing } \\
\text { supplements nor food intake of vitamin } \\
\text { B6, vitamin BI2 and riboflavin were } \\
\text { associated with lung cancer risk }\end{array}$ & $\begin{array}{l}\text { Study was designed to assess folic acid } \\
\text { from food or supplements; vitamin B6 } \\
\text { and BI2 intake from supplements was } \\
\text { not assessed }\end{array}$ \\
\hline Fanidi et al, $2019^{59}$ & $\begin{array}{l}\text { Case-control study } \\
\text { nested within LC3 } \\
\text { study, international }\end{array}$ & $\begin{array}{l}N=5,183 \text { case-control } \\
\text { pairs } \\
\text { Genetic data from } \\
N=29,266 \text { cases and } \\
N=56,450 \text { controls } \\
\text { from the TRICL- } \\
\text { ILCCO study }\end{array}$ & $\begin{array}{l}\text { Circulating vitamin } \mathrm{B} 12 \text { was positively } \\
\text { associated with overall lung cancer risk } \\
\text { in a dose-response fashion; findings } \\
\text { support the hypothesis that high vitamin } \\
\mathrm{B} 12 \text { status increases the risk of lung } \\
\text { cancer }\end{array}$ & $\begin{array}{l}\text { Study has several weaknesses: secondary } \\
\text { analysis of data from } 20 \text { cohorts that were } \\
\text { not designed to study such association; } \\
\text { reference groups of the different cohorts } \\
\text { were the ones with the lowest levels of } \\
\text { circulating vitamin BI2; BI2 not measured } \\
\text { but "estimated" in the TRICL-ILCCO } \\
\text { study }\end{array}$ \\
\hline Zuo et al, $2019^{81}$ & $\begin{array}{l}\text { Case control study } \\
\text { nested within LC3, } \\
\text { international }\end{array}$ & $\begin{array}{l}\mathrm{N}=5,232 \text { case-control } \\
\text { pairs }\end{array}$ & $\begin{array}{l}\text { Increased vitamin B6 catabolism related } \\
\text { to inflammation and immune activation } \\
\text { is associated with a higher risk of } \\
\text { developing lung cancer }\end{array}$ & $\begin{array}{l}\text { Conclusions are based on } \\
\text { measurements at a single time point } \\
\text { (regression dilution bias possible) }\end{array}$ \\
\hline Yang et al, $2018^{67}$ & $\begin{array}{l}\text { Meta-analysis of I4 } \\
\text { case-control studies/ } \\
\text { nested case-control } \\
\text { studies, international }\end{array}$ & $\begin{array}{l}\mathrm{N}=8,097 \text { lung cancer } \\
\text { patients and } \\
\mathrm{N}=10,008 \text { healthy } \\
\text { controls }\end{array}$ & $\begin{array}{l}\text { Serum folate and vitamin B6 might be } \\
\text { protective factors against lung } \\
\text { carcinogenesis and Hcy could contribute } \\
\text { to lung cancer risk }\end{array}$ & $\begin{array}{l}\text { Included cohorts were designed for such } \\
\text { analysis; blood samples were used } \\
\text { instead of intake estimates; three studies } \\
\text { with reliable evidence, II studies with } \\
\text { less certain evidence }\end{array}$ \\
\hline Zuo et al, $2018^{82}$ & $\begin{array}{l}\text { Nested case- } \\
\text { control study, } \\
\text { Europe }\end{array}$ & $\begin{array}{l}\mathrm{N}=892 \text { incident lung } \\
\text { cancer cases and } \\
\mathrm{N}=1,748 \text { controls } \\
\text { from the EPIC study } \\
\text { cohort }\end{array}$ & $\begin{array}{l}\text { Increased vitamin B6 catabolism is } \\
\text { independently associated with a higher } \\
\text { risk of future lung cancer }\end{array}$ & $\begin{array}{l}\text { Conclusions are based on measurements } \\
\text { at a single time point (regression dilution } \\
\text { bias possible); median time between } \\
\text { blood draw and lung cancer diagnoses was } \\
5.2 \text { years }\end{array}$ \\
\hline Fanidi et al, $2018^{64}$ & $\begin{array}{l}\text { Nested case- } \\
\text { control study, } \\
\text { international }\end{array}$ & $\begin{array}{l}\mathrm{N}=5,364 \text { incident } \\
\text { case-control pairs } \\
\text { ( } \mathrm{NCl} \text { cohorts) }\end{array}$ & $\begin{array}{l}\text { Participants with higher circulating } \\
\text { concentrations of vitamin B6 and folate } \\
\text { had a modestly decreased risk of lung } \\
\text { cancer risk overall }\end{array}$ & $\begin{array}{l}\text { Conclusions are based on measurements } \\
\text { at a single time point (regression dilution } \\
\text { bias possible); median time between } \\
\text { blood draw and lung cancer diagnoses was } \\
6.3 \text { years }\end{array}$ \\
\hline Brasky et al, $2017^{34}$ & $\begin{array}{l}\text { Prospective cohort } \\
\text { study, US }\end{array}$ & $\begin{array}{l}\mathrm{N}=77, \mathrm{I} \text { I8 participants } \\
\text { (VITAL cohort) }\end{array}$ & $\begin{array}{l}\text { Use of vitamin B6 and BI2 from } \\
\text { individual supplement sources, but not } \\
\text { from multivitamins, was associated with } \\
\text { a } 30-40 \% \text { increase in lung cancer risk } \\
\text { among men; no association in women }\end{array}$ & $\begin{array}{l}\text { Study has several limitations (see } \\
\text { Vitamin B6 and /or BI2 and risk of lung } \\
\text { cancer) }\end{array}$ \\
\hline Zuo et al, $2015^{83}$ & $\begin{array}{l}\text { Prospective cohort } \\
\text { study, Norway }\end{array}$ & $\begin{array}{l}\mathrm{N}=6,539 \text { participants } \\
\text { (Hordaland Health } \\
\text { Study) }\end{array}$ & $\begin{array}{l}\text { Associations of vitamin B6 with cancer may } \\
\text { be related to increased catabolism of } \\
\text { vitamin B6 captured by plasma PAr, in } \\
\text { particular for lung cancer where } \\
\text { inflammation may be largely involved in } \\
\text { carcinogenesis; the active B6 vitamer PLP } \\
\text { was not significantly associated with overall } \\
\text { cancer risk }\end{array}$ & $\begin{array}{l}\text { Study investigated certain types of } \\
\text { cancer (including but not limited to lung } \\
\text { cancer); findings based on relatively } \\
\text { small case numbers per cancer type; } \\
\text { included a comprehensive panel of } \\
\text { vitamin B6 markers }\end{array}$ \\
\hline $\begin{array}{l}\text { Tastekin et al, } \\
2015^{84}\end{array}$ & $\begin{array}{l}\text { Case-control study, } \\
\text { Turkey }\end{array}$ & $\begin{array}{l}\mathrm{N}=40 \text { males with lung } \\
\text { cancer, } \mathrm{N}=40 \text { healthy } \\
\text { controls }\end{array}$ & $\begin{array}{l}\text { Plasma folate levels significantly lower in } \\
\text { lung cancer cases compared with } \\
\text { controls }(P<0.00 \mathrm{I}) \text { but no significant } \\
\text { difference with regard to } B \mid 2 \text { levels }\end{array}$ & Small sample size \\
\hline
\end{tabular}


Table 3 (Continued).

\begin{tabular}{|c|c|c|c|c|}
\hline Reference & $\begin{array}{l}\text { Study Type and } \\
\text { Country }\end{array}$ & Sample Size & Main Findings & Comment \\
\hline Baltar et al, $2013^{85}$ & $\begin{array}{l}\text { Nested case- } \\
\text { control study, } \\
\text { Europe }\end{array}$ & $\begin{array}{l}N=891 \text { cases; } \\
N=1,747 \text { controls } \\
\text { (EPIC cohort) }\end{array}$ & $\begin{array}{l}\text { Structural equation modeling (including } \\
\text { vitamins } \mathrm{B} 2, \mathrm{~B} 6 \text {, folate, } \mathrm{B} \mid 2 \text { ) indicated } \\
\text { roles of methionine-Hcy metabolism and } \\
\text { immune activation in lung cancer risk; of } \\
\text { the measured B vitamins, only folate and } \\
\text { B6 were significantly }(P<0.0 I) \text { lower in } \\
\text { cases than controls; tobacco smoking } \\
\text { remained exposure with strongest } \\
\text { impact on lung cancer risk }\end{array}$ & $\begin{array}{l}\text { Authors emphasize that complex } \\
\text { pathways (OCM, folate cycle, and } \\
\text { immune system) rather than activities of } \\
\text { single vitamins or nutrients should be } \\
\text { considered in lung cancer carcinogenesis }\end{array}$ \\
\hline Takata et al, $2012^{86}$ & $\begin{array}{l}\text { Prospective cohort } \\
\text { study, China }\end{array}$ & $\begin{array}{l}\text { N=74,94I women } \\
\text { (Shanghai Women's } \\
\text { Health Study) }\end{array}$ & $\begin{array}{l}\text { Dietary riboflavin intake was inversely } \\
\text { associated with lung cancer risk while } \\
\text { intakes of other B vitamins (eg, B6, BI2) } \\
\text { were not associated }\end{array}$ & $\begin{array}{l}\text { Large study of female never-smokers } \\
\text { with lung cancer; population had lower } \\
\text { intakes than other populations; intakes } \\
\text { of B6 and BI } 2 \text { only estimated; no } \\
\text { information on serum levels }\end{array}$ \\
\hline $\begin{array}{l}\text { Bassett et al, } \\
2012^{87}\end{array}$ & $\begin{array}{l}\text { Prospective cohort } \\
\text { study, Australia }\end{array}$ & $\begin{array}{l}\mathrm{N}=41,514 \text { people } \\
\text { (Melbourne } \\
\text { Collaborative Cohort } \\
\text { Study) }\end{array}$ & $\begin{array}{l}\text { Little evidence of an association } \\
\text { between intake of B vitamins or } \\
\text { methionine and lung cancer risk; weak } \\
\text { inverse association between riboflavin } \\
\text { and lung cancer risk in current smokers } \\
\text { needs further investigation }\end{array}$ & $\begin{array}{l}\text { Information on intake (self-reporting } \\
\text { questionnaire) and potential } \\
\text { confounding variables was only collected } \\
\text { at baseline; no information on serum } \\
\text { levels }\end{array}$ \\
\hline $\begin{array}{l}\text { Johansson et al, } \\
2010^{65}\end{array}$ & $\begin{array}{l}\text { Case-control study, } \\
\text { Europe }\end{array}$ & $\begin{array}{l}\mathrm{N}=899 \text { lung cancer } \\
\text { cases; } \mathrm{N}=1,770 \\
\text { controls (EPIC) }\end{array}$ & $\begin{array}{l}\text { Serum levels of vitamin B6 and } \\
\text { methionine were inversely associated } \\
\text { with risk of lung cancer }\end{array}$ & $\begin{array}{l}\text { Measurements of serum levels only at } \\
\text { baseline (regression dilution bias } \\
\text { possible) }\end{array}$ \\
\hline Ebbing et al, $2009^{88}$ & $\begin{array}{l}\text { Combined data } \\
\text { from two RCTs } \\
\text { (NORVIT, } \\
\text { WENBIT), Norway }\end{array}$ & $\begin{array}{l}\mathrm{N}=6,837 \text { participants } \\
\text { with ischemic heart } \\
\text { disease }\end{array}$ & $\begin{array}{l}\text { While treatment with vitamin } \mathrm{B} 6 \text { alone } \\
\text { was not associated with any significant } \\
\text { effects, treatment with folic acid plus } \\
\text { vitamin } \mathrm{B} / 2 \text { was associated with } \\
\text { increased cancer outcomes and all-cause } \\
\text { mortality }\end{array}$ & $\begin{array}{l}\text { Study has limitations since both RCTs } \\
\text { originally were not designed or powered } \\
\text { to study lung cancer risk; authors point } \\
\text { out that observed associations were } \\
\text { confined only to serum folate, } \\
\text { suggesting that the adverse effects were } \\
\text { mediated by folate, not by vitamin BI2 }\end{array}$ \\
\hline Tsao et al, $2007^{89}$ & $\begin{array}{l}\text { Case-control study, } \\
\text { China }\end{array}$ & $\begin{array}{l}\mathrm{N}=27 \text { patients and } \\
\mathrm{N}=23 \text { controls }\end{array}$ & $\begin{array}{l}\text { RBC levels of vitamins } B 2 \text { and } B 6 \text { were } \\
\text { reduced in NSCLC patients }\end{array}$ & Small sample size \\
\hline $\begin{array}{l}\text { Hartman et al, } \\
200 I^{66}\end{array}$ & $\begin{array}{l}\text { Nested case- } \\
\text { control study, } \\
\text { Finland }\end{array}$ & $\begin{array}{l}\mathrm{N}=300 \text { male case- } \\
\text { control pairs }\end{array}$ & $\begin{array}{l}\text { No significant associations were seen } \\
\text { between serum folate, vitamin BI2 or } \\
\text { Hcy and lung cancer risk; significantly } \\
\text { lower risk of lung cancer among men } \\
\text { who had higher serum vitamin B6 levels }\end{array}$ & $\begin{array}{l}\text { Correlations between dietary intake and } \\
\text { serum markers of B vitamins were not } \\
\text { strong; many males had inadequate } \\
\text { serum levels of folate and B6 }\end{array}$ \\
\hline
\end{tabular}

Abbreviations: EPIC, European Prospective Investigation into Cancer and Nutrition; Hcy, homocysteine; LC3, Lung Cancer Cohort Consortium; NCl, National Cancer Institute; NORVIT, Norwegian Vitamin Trial; NSCLC, non-small cell lung cancer; OCM, one-carbon metabolism; PAr, 4-pyridoxic acid/(pyridoxal I pyridoxal 5'-phosphate) ratio; PLP, pyridoxal 5' phosphate; RBC, red blood cell; RCTs, randomized controlled trials; TRICL-ILCCO, Transdisciplinary Research Into Cancer of the Lung-International Lung Cancer Consortium; US, United States; VITAL, vitamins and Lifestyle; WENBIT, Western Norway B Vitamin Intervention Trial.

and B6 have a major impact on the Hcy metabolism as coenzymes $^{3}$ and lower Hcy levels, ${ }^{48,49}$ leading to the logical assumption that sufficiently high vitamin B6/B12 levels could theoretically help protect against low BMD or fractures. ${ }^{48}$ Although most research primarily sought to define a positive effect of $\mathrm{B}$ vitamins, a few recent articles propose an alternative assumption. ${ }^{33,50}$ Our literature searches revealed seven relevant studies on vitamin B6 and/or B12 and the risk of hip fracture: four cohort studies, one longitudinal follow-up study, one case-control study, and one secondary analysis of data from two RCTs (Table 2)..$^{33,-50-56}$ 
The recent prospective cohort study by Meyer et $\mathrm{al}^{33}$ claimed that a combined high intake of vitamin B6 $(\geq 35 \mathrm{mg} /$ day) and B12 ( $\geq 20 \mu \mathrm{g} /$ day) in postmenopausal women is associated with an increased hip fracture risk and suggests that vitamin supplements should be used cautiously. A possible biological explanation is that high pyridoxine doses might increase the risk of falling due to neurological symptoms, including reversible neuropathy, ataxia, and decreased muscle tone, as mentioned before. Another explanation may be related to accelerated bone loss induced by vitamin $\mathrm{B} 6$ by counteracting the modulating influence of estrogens on steroid receptors. ${ }^{51}$ However, current use of vitamin B6 was not associated with an increased fracture risk at any of the evaluated doses in the study (relative risk $[\mathrm{RR}]=1.12(95 \%$ confidence interval $[\mathrm{CI}]=0.95-1.31)),{ }^{33}$ suggesting that the slightly increased fracture risk is rather related to chronic $(>2$ years) than to current pyridoxine use. However, this study $^{33}$ also has numerous limitations, including the design as a cohort study, self-reporting of participants, high age of the study population, high confounding bias, etc. In addition, information is available only on vitamin intakes but not on blood/serum levels. ${ }^{33}$ Information on reasons for supplement intake is also lacking. ${ }^{33}$ As most supplements contain multivitamins, supplementation of vitamin B6 and/or B12 is likely to be taken with vitamin $\mathrm{D}$ and calcium. ${ }^{48}$ We assume that multivitamin supplements are predominantly prescribed to or voluntarily used by elderlies at risk of fractures, which means that supplement usage is normally driven by being at risk. We also believe that many other factors (eg, age, body mass index, physical activity, osteoporosis) may have contributed to the incidence of hip fractures and that the data are insufficient to provide evidence for a correlation of vitamin B6 intake and hip fracture risk. Most articles identified by us (Table 2) do not confirm this correlation, and several studies even found correlations between a higher vitamin B6 and/or B12 status and a lower risk of hip fracture and/or higher BMD. ${ }^{52-56}$ In agreement with a recent report of combined data from two $\mathrm{RCTs},{ }^{50}$ Meyer et $\mathrm{al}^{33}$ also found no association between vitamin $\mathrm{B} 12$ and an increased fracture risk $(\mathrm{RR}=1.25 ; 95 \%$ $\mathrm{CI}=0.98-1.58)$.

In summary, it cannot be assumed that the risk of hip fractures increases with vitamin B6/B12 intake levels per se but that it is rather modulated by a complex interplay of many factors such as calcium or D vitamin or metabolic and pharmacological factors. It would be a mistake in our opinion to change intake recommendations for (possibly otherwise required) higher vitamin B6/ B12 doses based on conclusions that do not take sufficient account of the complex processes.

\section{Vitamin B6 and/or BI2 and Risk of Lung Cancer}

The idea that B vitamins - in one way or another-play a role in carcinogenesis is not new. Indeed, in a population-based cohort study, higher plasma levels of cobalamin were associated with higher risk of cancer. ${ }^{57}$ Due to their antioxidant properties and their substantial participation in one-carbon metabolism (OCM) and, thus, the regulation of DNA methylation, an impact is almost undeniable (reviewed in Gruber ${ }^{58}$ ). However, when discussing whether B vitamins have cancer-protective or riskincreasing effects, it should be highlighted that OCM and other metabolic cycles involved in carcinogenesis are highly complex processes depending on a variety of interrelated factors. ${ }^{58}$ Nevertheless, several recent epidemiological studies do claim an association between lung cancer risk and B vitamins. ${ }^{34,59}$ In total, we identified nine casecontrol studies, four cohort studies, one meta-analysis of 14 case-control studies, and one analysis of combined data from two RCTs (Table 3) dealing with vitamin B6 and/or B12 and lung cancer.

One of the few studies proposing an association between $\mathrm{B}$ vitamins and lung cancer risk was published by Brasky et $\mathrm{al}^{34}$ in 2017. The Vitamins and Lifestyle (VITAL) cohort included 77,118 subjects aged 50-76 from the US and examined the association between the long-term use of supplemental B vitamins and lung cancer risk. ${ }^{34}$ In the 6-year follow-up on average, 808 lung cancer cases occurred. The 10-year average daily doses from individual and multivitamin supplements as well as mixtures were retrospectively documented at baseline by selfassessment questionnaires. When trying to establish associations, the authors found the long-term use of high-dose vitamin B6 (defined as $>20 \mathrm{mg} /$ day) or B12 (defined as $>55 \mu \mathrm{g}$ /day) from individual supplement sources, but not multivitamins, to be associated with a $30-40 \%$ increase in lung cancer risk among male smokers. ${ }^{34}$ However, in our opinion, the study does not prove causality between $B$ vitamin intake and lung cancer risk and has significant limitations, as already pointed out by Obeid and Pietrzik. ${ }^{60}$ When reviewing patient characteristics of the VITAL study, $42 \%$ of lung cancer cases were $\geq 70$ years, while 
only $19 \%$ of controls belonged to this age group. ${ }^{34}$ The percentage of smokers was almost four times higher for cases than controls. ${ }^{34}$ This imbalance was even more evident for smoking duration: $71 \%$ of lung cancer cases had smoked for $>35$ years vs $16 \%$ of controls. Likewise, $70 \%$ of cases and only $19 \%$ of controls had smoked $>25$ packyears of cigarettes. ${ }^{34}$ Finally, the history of COPD was 5 -times more frequent in cases than controls, ${ }^{34}$ and it is well known that this condition increases the risk of lung cancer - especially squamous cell carcinoma - by the factor five. ${ }^{61,62}$ Because these determinants of lung cancer, such as advanced age, cigarette smoking, and COPD history, were much more prevalent in cases than controls, ${ }^{34}$ it is much more likely that the authors estimated the effect of these well-known risk factors instead of the effect of B vitamins on this outcome. ${ }^{34,60}$

Without measuring serum levels over time, Brasky et $\mathrm{al}^{34}$ started from the premise that a self-reported intake above the RDA reflects disturbed vitamin homeostasis, but self-reported intakes should not be taken as surrogate markers for vitamin levels. As mentioned before, age, smoking status, smoking duration, smoking intensity, COPD history, and some genetic variants are well-known lung cancer risk factors. ${ }^{63}$ The fact that these factors were also associated with vitamin usage raises the suspicion that the association between vitamin intake and cancer was due to confounding, despite the authors' attempts to compensate for it through adjustment for known or suspected risk factors. Like Obeid and Pietrzik, ${ }^{60}$ we consider a reverse causality more likely; ie, knowing to have an increased risk (which every smoker does) can trigger healthier behaviors in other areas of life, including taking vitamin supplements. Therefore, we do not believe that the VITAL study uncovered a causal relationship between supplement intake and lung cancer. Apart from our own assumptions, many studies contradict the findings from a scientific point (Table 3). For example, several authors reported inverse relationships between vitamin B6 levels and lung cancer risk, ${ }^{64-66}$ and Yang et al ${ }^{67}$ even consider a possible protective effect of vitamin B6 in a systematic review and meta-analysis, including 14 studies and $>8,000$ patients. This protective effect of vitamin B6 has also been described in gastrointestinal cancer, pancreatic cancer, and breast cancer. ${ }^{68-70}$

Taken together, the current evidence from (almost exclusively epidemiological) studies on a relationship between vitamin B6 and/or B12 intake and lung cancer risk is not conclusive. ${ }^{34,-59,-64-67,80-89}$ The results of
Brasky et $\mathrm{al}^{34}$ regarding vitamins $\mathrm{B} 6$ and $\mathrm{B} 12$ should be interpreted with caution. The potential role of B vitamins in lung cancer carcinogenesis should be regarded in a bigger context and consider the complex interaction in OCM, folate cycle, etc., which we believe requires further intense research. However, the focus should still be on the actual high-risk factors such as smoking, which increase the risk many times. ${ }^{63}$

\section{Additional Considerations: General Morbi-Mortality}

Although the combination of vitamin B6 and B12 plus vitamin B9 (folic acid) has been suggested to prevent cardiovascular events (considering the relationship between Hcy levels and vascular risk), ${ }^{71,72}$ high doses of vitamin B6 (25 mg/day), vitamin B12 (1 mg/day), and vitamin B9 $(2.5 \mathrm{mg} /$ day) resulted in reduced glomerular filtration rates and increased vascular events in a placebo-controlled trial in patients with diabetic nephropathy. ${ }^{73}$ Correspondingly, higher vitamin B12 plasma levels were associated with a higher mortality risk in a cohort of hemodialysis patients. ${ }^{74}$ The same study found low serum folate levels to be related with the same higher risk of all-cause mortality, ${ }^{74}$ showing that overall evidence remains controversial and no final recommendation can be established. However, we suggest that levels of B vitamins should be anyway continuously monitored to reach adequate levels in patients with chronic kidney disease requiring supplements. Unlike patients with nephropathy, the frequency of adverse events in patients with cardiovascular disease using B vitamin supplements is similar to placebo. ${ }^{75}$

Interestingly, serum vitamin B12 levels may be increased in patients with heart failure, regardless of vitamin supplementation. ${ }^{76}$ This increase may be attributed to a subclinical liver dysfunction (cardiohepatic syndrome) which leads to the release of vitamin B12 from damaged liver cells and - paradoxically - produces clinical signs of vitamin B12 deficiency. ${ }^{76}$

Finally, some studies reported an association between higher plasma vitamin B12 levels and mortality in elderly $^{77}$ and hospitalized patients (without vitamin B12 intake). ${ }^{78}$ A recent longitudinal cohort study also showed this association in a general population of apparent nonusers of vitamin B12 supplements, ${ }^{79}$ but the authors did not establish a causal relationship due to residual confounding factors. ${ }^{79}$ 


\section{Limitations}

This review has several limitations. First, we have limited this review and the corresponding literature searches to various current safety aspects (ie, neurotoxicity, risk of hip fracture, and risk of lung cancer) and have not fully captured the entire evidence of the past decades in other areas. Our literature searches may therefore have been biased by our prior knowledge of the particularly highlighted epidemiologic cohort studies. Second, we assume that, despite the widespread use of B vitamins, the scientific literature does not fully reflect clinical reality. For example, underreporting of safety issues is a common problem, particularly in countries with less well-established health systems. ${ }^{90}$

\section{Conclusion}

The high-dose use of neurotropic B vitamins as medicinal products has proven useful in specific indications such as PN. We consider vitamin B1 as generally safe, with adverse reactions being limited to parenteral administration. If dosage recommendations especially for vitamin B6 are followed, we also consider the benefit-risk ratio to be advantageous. Ideally, treatment duration should be $<6$ months and serum levels monitored during treatment. This not only helps to avoid the rare cases of vitamin B6 overdose with potential neurological side-effects, but also to prevent possible undersupply, which can still occur with malabsorption despite high doses. Taking low doses of neurotropic B vitamins to prevent deficiencies in the otherwise healthy population is also well-established and can be considered safe overall. In our opinion, a causal relationship between the vitamin B6 and/or B12 intake and an increased hip fracture or lung cancer risk cannot be considered at present. We rather propose not to focus on individual nutrients, but to examine the complex interplay of the innumerable involved metabolic processes in detail and to expand the basic research on the development of these diseases on a biochemical level. Considering the predominantly renal excretion of these vitamins and a potentially increased risk of adverse outcomes in patients with diabetic nephropathy, we recommend monitoring B vitamin levels in this patient group.

\section{Acknowledgments}

Medical writing assistance was provided by Dittmann Medical Writing (Hamburg, Germany), the work was funded by P\&G Health International.

\section{Author Contributions}

All authors made substantial contributions to the conception and design, acquisition of data, or analysis and interpretation of data; took part in drafting the article or revising it critically for important intellectual content; agreed to submit to the current journal; gave final approval of the version to be published; and agree to be accountable for all aspects of the work.

\section{Disclosure}

The work was funded by $P \& G$ Health International. C. Calderon-Ospina holds consultative activities for $P \& G$ Health International and reports personal fees from them outside the submitted work. The authors report no other potential conflicts of interest for this work.

\section{References}

1. Zempleni J, Suttie JW, Gregory JF III, Stover PJ. Handbook of Vitamins. 5th ed. CRC Press; 2013.

2. Bender DA. B vitamins in the nervous system. Neurochem Int 1984;6(3):297-321. doi:10.1016/0197-0186(84)90072-X

3. Calderon-Ospina CA, Nava-Mesa MO. B Vitamins in the nervous system: current knowledge of the biochemical modes of action and synergies of thiamine, pyridoxine, and cobalamin. CNS Neurosci Ther. 2020;26(1):5-13. doi:10.1111/cns.13207

4. Yoon SM. Micronutrient deficiencies in inflammatory bowel disease: trivial or crucial? Intest Res. 2016;14(2):109-110. doi:10.5217/ ir.2016.14.2.109

5. Filippi J, Al-Jaouni R, Wiroth J-B, Hebuterne X, Schneider SM. Nutritional deficiencies in patients with Crohn's disease in remission. Inflamm Bowel Dis. 2006;12(3):185-191. doi:10.1097/01.MIB.0000 206541.15963.c3

6. Cook CCH, Hallwood PM, Thomson AD. B vitamin deficiency and neuropsychiatric syndromes in alcohol misuse. Alcohol Alcohol. 1998;33(4):317-336. doi:10.1093/oxfordjournals.alcalc.a008400

7. Adaikalakoteswari A, Rabbani N, Waspadji S, et al. Disturbance of B-vitamin status in people with type 2 diabetes in Indonesia-link to renal status, glycemic control and vascular inflammation. Diabetes Res Clin Pract. 2012;95(3):415-424. doi:10.1016/j.diabres.2011. 10.042

8. Nix WA, Zirwes R, Bangert V, et al. Vitamin B status in patients with type 2 diabetes mellitus with and without incipient nephropathy. Diabetes Res Clin Pract. 2015;107(1):157-165. doi:10.1016/j. diabres.2014.09.058

9. Kjeldby IK, Fosnes GS, Ligaarden SC, Farup PG. Vitamin B6 deficiency and diseases in elderly people-a study in nursing homes. $B M C$ Geriatr. 2013;13:13. doi:10.1186/1471-2318-13-13

10. Hung SC, Hung SH, Tarng DC, Yang WC, Chen TW, Huang TP. Thiamine deficiency and unexplained encephalopathy in hemodialysis and peritoneal dialysis patients. Am J Kidney Dis. 2001;38 (5):941-947. doi:10.1053/ajkd.2001.28578

11. Obeid R, Jung J, Falk J, et al. Serum vitamin B12 not reflecting vitamin B12 status in patients with type 2 diabetes. Biochimie. 2013;95(5):1056-1061. doi:10.1016/j.biochi.2012.10.028

12. Andres E, Loukili NH, Noel E, et al. Vitamin B12 (cobalamin) deficiency in elderly patients. CMAJ. 2004;171(3):251-259. doi:10. 1503/cmaj.1031155

13. O'Keeffe ST. Thiamine deficiency in elderly people. Age Ageing 2000;29(2):99-101. doi:10.1093/ageing/29.2.99 
14. Chiang E-P, Smith DE, Selhub J, Dallal G, Wang Y-C, Roubenoff R. Inflammation causes tissue-specific depletion of vitamin B6. Arthritis Res Ther. 2005;7(6):R1254-R1262. doi:10.1186/ar1821

15. Ortiz-Guerrero G, Amador-Munoz D, Calderon-Ospina CA, LopezFuentes D, Nava Mesa MO. Proton pump inhibitors and dementia: physiopathological mechanisms and clinical consequences. Neural Plast. 2018;2018:5257285. doi:10.1155/2018/5257285

16. Kim J, Ahn CW, Fang S, Lee HS, Park JS. Association between metformin dose and vitamin B12 deficiency in patients with type 2 diabetes. Medicine (Baltimore). 2019;98(46):e17918. doi:10.1097/ MD.000000000017918

17. Dierkes J, Westphal S. Effect of drugs on homocysteine concentrations. Semin Vasc Med. 2005;5(2):124-139. doi:10.1055/ s-2005-872398

18. Head KA. Peripheral neuropathy: pathogenic mechanisms and alternative therapies. Altern Med Rev. 2006;11(4):294-329.

19. Hakim M, Kurniani N, Pinzon RT, et al. Management of peripheral neuropathy symptoms with a fixed dose combination of high-dose vitamin B1, B6 and B12: A 12-week prospective non-interventional study in Indonesia. Asian J Med Sci. 2018;9(1):32-40. doi:10.3126/ ajms.v9i1.18510

20. Sriram K, Manzanares W, Joseph K. Thiamine in nutrition therapy. Nutr Clin Pract. 2012;27(1):41-50. doi:10.1177/0884533611426149

21. Spinneker A, Sola R, Lemmen V, Castillo MJ, Pietrzik K, GonzalezGross M. Vitamin B6 status, deficiency and its consequences-an overview. Nutr Hosp. 2007;22(1):7-24.

22. Kumar N. Neurologic aspects of cobalamin (B12) deficiency. Handb Clin Neurol. 2014;120:915-926. doi:10.1016/B978-0-7020-40870.00060-7

23. Stabler SP. Clinical practice. Vitamin B12 deficiency. $N$ Engl J Med. 2013;368(2):149-160. doi:10.1056/NEJMcp1113996

24. Geller M, Oliveira L, Nigri R, et al. B Vitamins for neuropathy and neuropathic pain. Vitam Miner. 2017;6:2. doi:10.4172/2376-1318. 1000161

25. Veraldi S, Benardon S, Diani M, Barbareschi M. Acneiform eruptions caused by vitamin B12: a report of five cases and review of the literature. J Cosmet Dermatol. 2018;17(1):112-115. doi:10.1111/jocd.12360

26. Tolerable upper intake levels for vitamins and minerals. European Food Safety Authority; 2006.

27. Morales-Gutierrez J, Diaz-Cortes S, Montoya-Giraldo MA, Zuluaga AF. Toxicity induced by multiple high doses of vitamin B12 during pernicious anemia treatment: a case report. Clin Toxicol (Phila). 2020;58:129-131. doi:10.1080/15563650.2019.1606432

28. Martinez de Espronceda Ezquerro I, Oscoz Jaime S, Yanguas Bayona JI. Vitamin B12-induced acne. Med Clin (Barc). 2018;151 (3):e15. doi:10.1016/j.medcli.2018.01.002

29. Balta I, Ozuguz P. Vitamin B12-induced acneiform eruption. Cutan Ocul Toxicol. 2014;33(2):94-95. doi:10.3109/15569527.2013.808657

30. Okereke OI, Cook NR, Albert CM, Van Denburgh M, Buring JE, Manson JE. Effect of long-term supplementation with folic acid and $\mathrm{B}$ vitamins on risk of depression in older women. $\mathrm{Br} J$ Psychiatry. 2015;206(4):324-331. doi:10.1192/bjp.bp.114.148361

31. McMahon JA, Green TJ, Skeaff CM, Knight RG, Mann JI, Williams SM. A controlled trial of homocysteine lowering and cognitive performance. $N$ Engl J Med. 2006;354(26):2764-2772. doi:10.1056/NEJMoa054025

32. Jerneren F, Elshorbagy AK, Oulhaj A, Smith SM, Refsum H, Smith AD. Brain atrophy in cognitively impaired elderly: the importance of long-chain omega-3 fatty acids and B vitamin status in a randomized controlled trial. Am J Clin Nutr. 2015;102 (1):215-221. doi:10.3945/ajcn.114.103283

33. Meyer HE, Willett WC, Fung TT, Holvik K, Feskanich D. Association of high intakes of vitamins B6 and B12 from food and supplements with risk of hip fracture among postmenopausal women in the nurses' health study. JAMA Netw open. 2019;2(5):e193591. doi:10.1001/jamanetworkopen.2019.3591
34. Brasky TM, White E, Chen C-L. Long-term, supplemental, one-carbon metabolism-related Vitamin B use in relation to lung cancer risk in the vitamins and lifestyle (VITAL) Cohort. $J$ Clin Oncol. 2017;35(30):3440-3448. doi:10.1200/JCO.2017.72.7735

35. Dietary Reference Intakes for Thiamin, Riboflavin, Niacin, Vitamin B6, Folate, Vitamin B12, Pantothenic Acid, Biotin, and Choline. Institute of Medicine (US) Standing Committee on the Scientific Evaluation of Dietary Reference Intakes and its Panel on Folate, Other B Vitamins, and Choline. Washington (DC): National Academies Press (US); 1998.

36. McLaughlin K, Joyal K, Lee S, et al. Safety of intravenous push thiamine administration at a tertiary academic medical center. J Am Pharm Assoc. 2020:pii: S1544-3191(19)30544-8. doi:10.1016/j.japh.2019.12.005.

37. Tjugum SL, Hedrick TL, Jean SJ, Heeney SA, Rohde KA, CampbellBright SL. Evaluation of the safety of intravenous thiamine administration in a large academic medical center. $J$ Pharm Pract. 2019;897190019872584. doi:10.1177/0897190019872584

38. Rao DB, Jortner BS, Sills RC. Animal models of peripheral neuropathy due to environmental toxicants. ILAR J. 2014;54(3):315-323. doi:10.1093/ilar/ilt058

39. Echaniz-Laguna A, Mourot-Cottet R, Noel E, Chanson J-B. Regressive pyridoxine-induced sensory neuronopathy in a patient with homocystinuria. BMJ Case Rep. 2018;2018:pii: bcr-2018225059. doi: $10.1136 / \mathrm{bcr}-2018-225059$

40. Berger AR, Schaumburg HH, Schroeder C, Apfel S, Reynolds R. Dose response, coasting, and differential fiber vulnerability in human toxic neuropathy: a prospective study of pyridoxine neurotoxicity. Neurology. 1992;42(7):1367-1370. doi:10.1212/wnl.42.7.1367

41. Dalton K, Dalton MJ. Characteristics of pyridoxine overdose neuropathy syndrome. Acta Neurol Scand. 1987;76(1):8-11. doi:10.1111/ j.1600-0404.1987.tb03536.x

42. van Hunsel F, van de Koppel S, van Puijenbroek E, Kant A. Vitamin B6 in health supplements and neuropathy: case series assessment of spontaneously reported cases. Drug Saf. 2018;41(9):859-869. doi:10.1007/s40264-018-0664-0

43. Kulkantrakorn K. Pyridoxine-induced sensory ataxic neuronopathy and neuropathy: revisited. Neurol Sci. 2014;35(11):1827-1830. doi:10.1007/s10072-014-1902-6

44. Mikalunas V, Fitzgerald K, Rubin H, McCarthy R, Craig RM. Abnormal vitamin levels in patients receiving home total parenteral nutrition. J Clin Gastroenterol. 2001;33(5):393-396. doi:10.1097/ 00004836-200111000-00010

45. Albin RL, Albers JW, Greenberg HS, et al. Acute sensory neuropathy-neuronopathy from pyridoxine overdose. Neurology. 1987;37(11):1729-1732. doi:10.1212/wnl.37.11.1729

46. Parry GJ, Bredesen DE. Sensory neuropathy with low-dose pyridoxine. Neurology. 1985;35(10):1466-1468. doi:10.1212/wnl.35.10.1466

47. Schaumburg H, Kaplan J, Windebank A, et al. Sensory neuropathy from pyridoxine abuse. A new megavitamin syndrome. $N$ Engl J Med. 1983;309(8):445-448. doi:10.1056/NEJM198308253090801

48. Fratoni V, Brandi ML. B vitamins, homocysteine and bone health. Nutrients. 2015;7(4):2176-2192. doi:10.3390/nu7042176

49. Swart KMA, van Schoor NM, Lips P. Vitamin B12, folic acid, and bone. Curr Osteoporos Rep. 2013;11(3):213-218. doi:10.1007/ s11914-013-0155-2

50. Garcia Lopez M, Bonaa KH, Ebbing M, et al. B Vitamins and hip fracture: secondary analyses and extended follow-up of two large randomized controlled trials. J Bone Miner Res. 2017;32 (10):1981-1989. doi:10.1002/jbmr.3189

51. Allgood VE, Cidlowski JA. Vitamin B6 modulates transcriptional activation by multiple members of the steroid hormone receptor superfamily. J Biol Chem. 1992;267(6):3819-3824.

52. Dai Z, Wang R, Ang LW, Yuan J-M, Koh W-P. Dietary B vitamin intake and risk of hip fracture: the Singapore Chinese Health Study. Osteoporos Int. 2013;24(7):2049-2059. doi:10.1007/s00198-0122233-1 
53. McLean RR, Jacques PF, Selhub J, et al. Plasma B vitamins, homocysteine, and their relation with bone loss and hip fracture in elderly men and women. J Clin Endocrinol Metab. 2008;93(6):2206-2212. doi: $10.1210 /$ jc. $2007-2710$

54. Gjesdal CG, Vollset SE, Ueland PM, Refsum H, Meyer HE, Tell GS. Plasma homocysteine, folate, and vitamin B 12 and the risk of hip fracture: the hordaland homocysteine study. J Bone Miner Res. 2007;22(5):747-756. doi:10.1359/jbmr.070210

55. Morris MS, Jacques PF, Selhub J. Relation between homocysteine and B-vitamin status indicators and bone mineral density in older Americans. Bone. 2005;37(2):234-242. doi:10.1016/j.bone.2005.04.017

56. Lumbers M, New SA, Gibson S, Murphy MC. Nutritional status in elderly female hip fracture patients: comparison with an age-matched home living group attending day centres. Br J Nutr. 2001;85 (6):733-740. doi:10.1079/bjn2001350

57. Arendt JFB, Pedersen L, Nexo E, Sorensen HT. Elevated plasma vitamin B12 levels as a marker for cancer: a population-based cohort study. J Natl Cancer Inst. 2013;105(23):1799-1805. doi:10.1093/ jnci/djt315

58. Gruber BM. B-Group Vitamins: chemoprevention? Adv Clin Exp Med. 2016;25(3):561-568. doi:10.17219/acem/33847

59. Fanidi A, Carreras-Torres R, Larose TL, et al. Is high vitamin B12 status a cause of lung cancer? Int J Cancer. 2019;145(6):1499-1503. doi:10.1002/ijc.32033

60. Obeid R, Pietrzik K. Smoking, B Vitamins, and lung cancer: the chicken or the egg causality dilemma. J Clin Oncol. 2018;36 (6):626-627. doi:10.1200/JCO.2017.75.9472

61. Durham AL, Adcock IM. The relationship between COPD and lung cancer. Lung Cancer. 2015;90(2):121-127. doi:10.1016/j.lungcan.20 15.08.017

62. Young RP, Hopkins RJ. Link between COPD and lung cancer. Respir Med. 2010;104(5):758-759. doi:10.1016/j.rmed.2009.11.025

63. Gibbons DL, Byers LA, Kurie JM. Smoking, p53 mutation, and lung cancer. Mol Cancer Res. 2014;12(1):3-13. doi:10.1158/1541-7786. MCR-13-0539

64. Fanidi A, Muller DC, Yuan J-M, et al. Circulating Folate, Vitamin B6, and methionine in relation to lung cancer risk in the lung cancer cohort consortium (LC3). J Natl Cancer Inst. 2018;110(1):djx119. doi:10.1093/jnci/djx119

65. Johansson M, Relton C, Ueland PM, et al. Serum B vitamin levels and risk of lung cancer. JAMA. 2010;303(23):2377-2385. doi:10.10 01/jama.2010.808

66. Hartman TJ, Woodson K, Stolzenberg-Solomon R, et al. Association of the B-vitamins pyridoxal 5'-phosphate $(\mathrm{B}(6)), \mathrm{B}(12)$, and folate with lung cancer risk in older men. Am J Epidemiol. 2001;153 (7):688-694. doi:10.1093/aje/153.7.688

67. Yang J, Li H, Deng H, Wang Z. Association of one-carbon metabolism-related vitamins (Folate, B6, B12), homocysteine and methionine with the risk of lung cancer: systematic review and meta-analysis. Front Oncol. 2018;8:493. doi:10.3389/fonc.2018.00493

68. Gylling B, Myte R, Schneede J, et al. Vitamin B-6 and colorectal cancer risk: a prospective population-based study using 3 distinct plasma markers of vitamin B-6 status. Am J Clin Nutr. 2017;105 (4):897-904. doi:10.3945/ajcn.116.139337

69. Huang JY, Butler LM, Midttun O, et al. Serum B6 vitamers (pyridoxal 5'phosphate, pyridoxal, and 4-pyridoxic acid) and pancreatic cancer risk: two nested case-control studies in Asian populations. Cancer Causes Control. 2016;27(12):1447-1456. doi:10.1007/s10552-016-0822-6

70. Wu W, Kang S, Zhang D. Association of vitamin B6, vitamin B12 and methionine with risk of breast cancer: a dose-response meta-analysis. Br J Cancer. 2013;109(7):1926-1944. doi:10.1038/ bjc. 2013.438

71. Stanger O, Herrmann W, Pietrzik K, et al. Clinical use and rational management of homocysteine, folic acid, and B vitamins in cardiovascular and thrombotic diseases. Z Kardiol. 2004;93(6):439-453. doi:10.1007/s00392-004-0075-3
72. Clarke R, Armitage J. Vitamin supplements and cardiovascular risk: review of the randomized trials of homocysteine-lowering vitamin supplements. Semin Thromb Hemost. 2000;26(3):341-348. doi:10. 1055/s-2000-8101

73. House AA, Eliasziw M, Cattran DC, et al. Effect of B-vitamin therapy on progression of diabetic nephropathy: a randomized controlled trial. JAMA. 2010;303(16):1603-1609. doi:10.1001/jama.20 10.490

74. Soohoo M, Ahmadi S-F, Qader H, et al. Association of serum vitamin B12 and folate with mortality in incident hemodialysis patients. Nephrol Dial Transplant. 2017;32(6):1024-1032. doi:10.1093/ndt/ gfw090

75. Ebbing M, Bleie O, Ueland PM, et al. Mortality and cardiovascular events in patients treated with homocysteine-lowering $\mathrm{B}$ vitamins after coronary angiography: a randomized controlled trial. JAMA. 2008;300(7):795-804. doi:10.1001/jama.300.7.795

76. Argan O, Ural D, Karauzum K, et al. Elevated levels of vitamin B12 in chronic stable heart failure: a marker for subclinical liver damage and impaired prognosis. Ther Clin Risk Manag. 2018;14:1067-1073. doi:10.2147/TCRM.S164200

77. Mendonca N, Jagger C, Granic A, et al. Elevated Total homocysteine in all participants and plasma vitamin B12 concentrations in women are associated with all-cause and cardiovascular mortality in the very old: the Newcastle 85+ study. J Gerontol a Biol Sci Med Sci. 2018;73 (9):1258-1264. doi:10.1093/gerona/gly035

78. Cappello S, Cereda E, Rondanelli M, et al. Elevated plasma vitamin B12 concentrations are independent predictors of in-hospital mortality in adult patients at nutritional risk. Nutrients. 2017;9:1. doi:10. 3390/nu9010001

79. Flores-Guerrero JL, Minovic I, Groothof D, et al. Association of plasma concentration of vitamin B12 with all-cause mortality in the general population in the Netherlands. JAMA Netw open. 2020;3(1): e1919274. doi:10.1001/jamanetworkopen.2019.19274

80. Takata Y, Shu X-O, Buchowski MS, et al. Food intake of folate, folic acid and other B vitamins with lung cancer risk in a low-income population in the Southeastern United States. Eur J Nutr. 2020;59:671-683. doi:10.1007/s00394-019-01934-5

81. Zuo H, Ueland PM, Midttun O, et al. Vitamin B6 catabolism and lung cancer risk: results from the Lung Cancer Cohort Consortium (LC3). Ann Oncol. 2019;30(3):478-485. doi:10.1093/annonc/mdz002

82. Zuo H, Ueland PM, Midttun O, et al. Results from the European prospective investigation into cancer and nutrition link vitamin B6 catabolism and lung cancer risk. Cancer Res. 2018;78(1):302-308. doi:10.1158/0008-5472.CAN-17-1923

83. Zuo H, Ueland PM, Eussen SJPM, et al. Markers of vitamin B6 status and metabolism as predictors of incident cancer: the Hordaland Health Study. Int J Cancer. 2015;136(12):2932-2939. doi:10.1002/ ijc. 29345

84. Tastekin D, Erturk K, Bozbey HU, et al. Plasma homocysteine, folate and vitamin B12 levels in patients with lung cancer. Exp Oncol. 2015;37(3):218-222. doi:10.31768/2312-8852.2015.37(3):2 18-222

85. Baltar VT, Xun WW, Johansson M, et al. A structural equation modelling approach to explore the role of $\mathrm{B}$ vitamins and immune markers in lung cancer risk. Eur J Epidemiol. 2013;28(8):677-688. doi:10.1007/s10654-013-9793-z

86. Takata Y, Cai Q, Beeghly-Fadiel A, et al. Dietary B vitamin and methionine intakes and lung cancer risk among female never smokers in China. Cancer Causes Control. 2012;23(12):1965-1975. doi:10.10 07/s10552-012-0074-z

87. Bassett JK, Hodge AM, English DR, et al. Dietary intake of $\mathrm{B}$ vitamins and methionine and risk of lung cancer. Eur J Clin Nutr. 2012;66(2):182-187. doi:10.1038/ejcn.2011.157

88. Ebbing M, Bonaa KH, Nygard O, et al. Cancer incidence and mortality after treatment with folic acid and vitamin B12. JAMA. 2009;302(19):2119-2126. doi:10.1001/jama.2009.1622 
89. Tsao S-M, Yin M-C, Liu W-H. Oxidant stress and B vitamins status in patients with non-small cell lung cancer. Nutr Cancer. 2007;59(1):8-13. doi:10.1080/016355807013 65043
90. Aagaard L, Strandell J, Melskens L, Petersen PS, Holme Hansen E. Global patterns of adverse drug reactions over a decade: analyses of spontaneous reports to VigiBase ${ }^{\mathrm{TM}}$. Drug Saf. 2012;35(12):11 71-1182. doi:10.1007/BF03262002

\section{Publish your work in this journal}

Therapeutics and Clinical Risk Management is an international, peerreviewed journal of clinical therapeutics and risk management, focusing on concise rapid reporting of clinical studies in all therapeutic areas, outcomes, safety, and programs for the effective, safe, and sustained use of medicines. This journal is indexed on PubMed Central, CAS,
EMBase, Scopus and the Elsevier Bibliographic databases. The manuscript management system is completely online and includes a very quick and fair peer-review system, which is all easy to use. Visit http://www.dovepress.com/testimonials.php to read real quotes from published authors. 